\title{
Palliative nursing care as applied to geriatric: An integrative literature review
}

\author{
Jefferson Garcia Guerrero* \\ Department of Nursing, Fakeeh College for Medical Sciences, Al Hamra District, Jeddah, Saudi Arabia
}

\begin{abstract}
The objective of the integrative literature review is to describe and explore the current status of research evidences about palliative care as applied to geriatric population and in the nursing context.

Methods: Integrative review was conducted to determine current knowledge of the chosen topic. MEDLINE, PubMed and CINAHL online databases or systematic engine search were utilized to obtain the research articles relevant to this integrative review. The data consisted of 31 research articles about palliative care nursing.

Results: This integrative literature review revealed at least four general themes emerged in this study. Such major theme includes basic concepts of palliative care nursing, palliative care assessment, fundamental palliative care skills, and the palliative care team.
\end{abstract}

Conclusion and recommendation: The study concluded that there are only limited research findings is currently available about palliative care nursing. Therefore, future research is needed to gather information, further validity and research evidences about this emerging discipline in nursing.

\section{Introduction}

Currently, aged 60 or over was about $10 \%$ of the world's population was. On 2025, it was projected that the figure will escalate to $15 \%$ and by year 2050, the figure will increase to $21 \%$ [1]. This recent demographic data reveals a growth in the number of the world's elder population [2]. Unfortunately, the number of individuals with chronic diseases is increasing due to advance an aging society $[3,4]$. The prevalence of lifethreatening diseases increases the likelihood of a prolonged period of chronic illness prior to death [5]. Thus, the rapidly expanding population of older adults predisposes them to certain disease [6]. The older adult, especially those 75 years old, experiences multiple morbidities and complications. It was even cited that elder adults have developed rates of persistent disorder. Based from the statistics, more than $70 \%$ having chronic cardiovascular conditions, whereas the remaining $30 \%$ have chronic respiratory, gastrointestinal, endocrine, musculoskeletal and nervous system disorders [1]. Specifically, ischemic heart diseases, cancer, stroke, and respiratory failure are the foremost source of death in developing countries. While terminal cancer, cardiac, respiratory and renal failure are the conditions with an inevitable fatal outcome [7].

The increase prevalence of multi-morbidity rises with age [3]. Nonetheless, the utmost functional deterioration initiated by aging is deemed as a normal biological process [1]. Physical aging is the product of degeneration of multiple organ systems from numerous diseases and several risk factors. Such degeneration leads to frailty, functional limitation, physical impairment, cognitive disability, impaired mobility, and disability [8]. Averting elder individuals from incapacity has been recognized as one of the paramount approaches for attaining healthy aging [1]. Patients' families conveyed that patients were in moderate to severe pain with reports of 50\% [9]. Reports even cited that there is now a demographic shift and an increasing incidence of serious progressive chronic diseases resulting to early death while other illnesses resulting to the likelihood of slow death $[10,11]$. Furthermore, studies showed that $40 \%$ of patient death were reported at least 10 days in intensive care units of hospitals [9]. Thus, the majority of all patient deaths occurred in healthcare institutions [7]. Unfortunately, trainees, caregivers, nurses and physicians feel underprepared to provide end-of-life care skills and lack fundamental training in palliative care [12]. Thus, it is essential to deliver more elderly with quality and comprehensive healthcare. Therefore, specialized knowledge, attitude, and skills are prerequisite to deliver palliative care to older people facing life-threatening illness [2].

As currently the leading cause of death worldwide, an ageing population and chronic illnesses confront existing meeting the healthcare requirements of elder individuals. End-of-life care provision for elder individuals in health care [13]. This is a challenge for nurses because the last phase of life can lead to significant effect in the patient's life before death [11]. The palliative care necessities of chronically ill patients have been gradually acknowledged but the approach to palliative care remains inadequate and differs from area to area [14].

\section{Methods}

Integrative review was conducted to determine current knowledge of the chosen topic. MEDLINE, PubMed and CINAHL online databases or systematic engine search were utilized to obtain the research articles relevant to this integrative review. The data consisted of 31 research articles about palliative care nursing. Thus, 31 eligible studies were identified. The researcher used content analysis as a qualitative approach

${ }^{\star}$ Correspondence to: Jefferson Garcia Guerrero, Department of Nursing, Fakeeh College for Medical Sciences, Al Hamra District, Jeddah, Saudi Arabia, Tel: 966570302970, E-mail: jgguerrero@fcms.edu.sa

Key words: palliative care nursing, geriatric, end-of-life care, quality of life

Received: February 08, 2019; Accepted: March 18, 2019; Published: March 21 , 2019 
in reviewing the articles. Content analysis is a research method for studying social phenomena using the available documents, artifact and literature to examine the communication pattern in a systematic manner/way.

Inclusion criteria of the study includes the following: a) All selected articles must be written in the English language; b) All articles must be published in peer-reviewed journals; c) All articles must be published from at least 15 years from now (2003-2018); d) All articles must contain at least one of the following keywords palliative care nursing, palliative care skills, assessment in palliative care, the palliative care team.

Data analyses were performed from October 10, 2018 to December $5,2018$.

\section{Results and discussion}

This integrative literature review revealed at least four general themes emerged in this study. Such major theme includes basic concepts of palliative care nursing, palliative care assessment, fundamental palliative care skills, and the palliative care team. First major theme is the basic concepts of palliative care nursing. This comprised of definition, characteristics, principles, levels, approaches, and philosophy. For palliative care assessment, the following subthemes were generated: physical, psychological, sociocultural, spiritual, and family support. Third, basic/fundamental palliative care skills emerged from this literature review, namely: symptom management, pain control, communication skills, supportive care, advance care planning, and end-of-life care. Last major theme emerged is the palliative care team which comprises the interdisciplinary and multidisciplinary team like physicians, nurses, practitioners, social workers, pastors/ priest/chaplain, a pharmacist, advance practice nurses, gerontologists, a physical therapist, occupational therapists, physiotherapist, a psychiatrist, and dieticians among others.

\section{Basic concepts in palliative care nursing}

The World Health Organization (WHO) delineates palliative care as a method which recuperates the quality of life of patients and their families confronting life-threatening illness, through the avoidance, evaluation, and management of pain and other physical, psychosocial and spiritual difficulties $[15,16]$.

As promulgated by WHO, palliative care encompasses the following principles: in managing sufferings, controlling symptoms, promoting spiritual well-being, offering the best quality of life, reducing worthless medical management, and preventing prolong pain for terminally ill patients [17].

Palliative care is a distinct medical specialty that address an essential dispute of suffering, relief of suffering, symptomatic relief, and spiritual care [18].

Furthermore, the following are five principles of palliative care: a) Palliative care respects the goals, likes and choices of the dying person; b) Palliative care looks after the medical, emotional, social, and spiritual needs of the dying person; c) Palliative care supports the needs of family members. The need to deliver outstanding end of life care; d) Safe-guard human dignity and to promote better care of the dying; e) Must address the right-to-die issues and right to end-of-life decision making [19].

Palliative care as an approach as partaking the subsequent characteristics: upholds life and favors dying as a normal process; neither accelerates nor adjourns death; delivers liberation from pain and other stressful symptoms; assimilates the psychological and spiritual facets of patient care; offers a support system to benefit patients live as dynamically as possible until death; deals a support system to assist the family manage during the patient's illness and during their own bereavement; discourse the necessities of clients and families including bereavement process; enhance quality of life; offer therapies intended to prolong life; supports death with dignity; and cope afflicting clinical complications [18]

Three levels of palliative care: primary (primary responsibility of nurses, improve quality of care, relief of patient's suffering), secondary (provided by hospital specialist, hospice consultant), and tertiary (academic, educational, research medical centers) [9].

Palliative care, on the other hand, as an integrated care approach for person with multi-morbidity necessitates integration and synchronization of care. Integrated care is the organization and conveyance of health services in a continuum of long-term care, rehabilitation, and palliative care services. The following are five elements of integrated care for multi-morbidity, namely: comprehensive holistic needs assessment, person-centered care, selfmanagement, integration coordinated care services, and collaboration. In this connection, multidisciplinary collaborative relationship among healthcare providers in the field of gerontology, geriatrics, and palliative care to provide excellent delivery of palliative care for elderly dying people [20].

Palliative care has the philosophy of caring for the terminally ill emphasizes that the patient and family live each day as fully as possible [16]. Basic principle is not to restrict patient on the last days or hours of life [11]. Palliative care provides end-of-life care that is care offered in the final weeks before death by providing comprehensive care in the final year, months, and weeks of life of terminally ill patients [5]. Palliative care is any management which does not have the aim of curative care. Patient's goals are to patient die peacefully through the natural course of the disease. It is often misconstrued as "giving up" or "hastening end of life. True palliation means specifying comfort which optimizes quality-of-life care [21]. Palliative care is important to patients who are diagnosed with advance stages of disease when treatment is no longer effective [22]. It allows transition approach from cure to care to patients experiencing life-threatening illness and fatal diseases [22]. There is a paradigm shift that palliative care has shifted from curative goals to comfort-focused care, person-centered and carefocused perspective because of the belief that there is nothing can be done during late-stage illness for patients approaching the end of life [5]. It also centers on sustenance and care of the dying person and family with the goal of easing a peaceful and dignified death. It stresses on holistic care to improve the quality of life rather than cure [15]. It is a holistic approach toward, health, illness and death [16]. Thus, it means initiating care holistically to person that includes body, mind, spirit, heart and soul. It believes that dying is natural and personal [19].

Nurses have significant effect in patients live until they die. Nursing has a long history of providing holistic, patient-centered, and family-centered care. Nursing is important because it cares for dying patients. Knowledge about end-of-life principles of care are important. Patient and family needs at the end of life are not well understood and not appreciated. Nurses must support the patient and family throughout the expiring process and grief. This involves a symptom control and pain management [15]. Lastly, Nurses must reflect their practice and must ensure to provide the appropriate and highest quality of end-of-life care [11]. 


\section{Palliative care as an emerging nursing discipline/specialty}

Previously, lack of palliative care education in the undergraduate program has been recognized as an impediment to the improvement of discipline [23].

Palliative aged care is becomingly developed into a new specialty area and young scientific discipline [20,22]. Palliative care as a rapidly growing medical specialty has formed own distinctive and multifaceted knowledge and practice. In fact, the number of hospice palliative carerelated publications augmented promptly from 1993 to 2013 in the world. The improvement of palliative care in practice is evident through collective numbers of hospice palliative beds, mounting consumption of hospital hospice bed and home hospice care [20]. Likewise, the development of hospital-wide palliative care beds, clustered palliative care units, palliative care consultation services are all needed to improve care of the dying [5].

Specialized training, fundamental knowledge and basic skills in palliative care are indispensable for all health professionals who deal with patients in the final stages of their lives. Furthermore, the need to prepare and educate future professionals to deal with the necessities of patients and relatives facing life-threatening illnesses. There is a pressing necessity to incorporate education on the principles of palliative care in both undergraduate and graduate health care courses [22]. Ultimately, the need to provide high-quality palliative care needs of specialized populations is imperative [5].

\section{Assessment in palliative care nursing}

Palliative care expands the quality of death and dying for patients [9]. A palliative care plan includes care goals, family support, spiritual care, financial support, rehabilitative care plans, and comorbid disease management [16]. It also involves advance care planning, financial planning, symptom management. Factors affecting palliative aged care comprise illness/disease trajectory, symptom presentation, pharmacologic, physiologic changes, and psychological changes [24]. In addition, there is a need for interdisciplinary approach and multidimensional assessment in terms of: symptom management, comorbid conditions, subsequent deterioration, death planning, functional status, social worker referral, support network, community services, bereavement services, and financial situation. All of which are integral parts of the delivery of palliative care $[9,25]$. Therefore, nurses must identify and address the physical, psychological, emotional, behavioral, social, cultural and spiritual needs of a person approaching death and his/her family $[11,26]$. Thus, the nurse must learn to prioritize and manage physical, psychological, spiritual, and the social aspect of care the dying patient $[24,27]$. In addition, another important nursing role includes coordinating care across all disciplines that fosters dying patients and family overall well-being [16].

Progressive disease, multiple co-morbidities, general physical deterioration, growing dependency, and diminishing activity are all indicators that a person may be resembling the end of life [13]. However, patients with chronic diseases frequently have complex comorbidities and high symptom burdens [28]. Multi-morbidity is the occurrence of two or more chronic conditions within the same person. Multimorbidity remains a challenge in the healthcare organization because the care provided by multiple care providers for persons with multiple chronic condition often lacks alignment [3]. Multiple comorbidities contribute to frailty. Thus, the probability of patient deterioration and mortality should be anticipated in the frail elder populace obtaining care in health care settings [13]. Nurse must incorporate and should have thoughtful consideration of comorbidities, frailty, and functional disabilities of the terminally ill patient. [1] Comorbid disease management involves monitoring for coexisting illness, preexisting medical condition, and increasing multiple comorbidities [16]. Likewise, the nurse must address and recognize the specific needs of a patient who shows exacerbation, chronic diseases and subsequent deteriorations, and dying trajectories [24]. It must coordinate care with other health care specialists [16]. Delay in recognizing that a patient may be deteriorating towards death consequences in delays of provision and assessment of palliative care and delays initiation of suitable endof-life care to patients [13].

The nurse must also address and meet the physical and physiologic needs of a dying patient. Interventions must focus on airway, bathing, personal hygiene, physical mobility, movement, nutrition, hydration, constipation, urinary elimination, sensory changes [15]. Likewise, the need to maintain functional support is done so a patient can return to performing activities of daily living [16].

The nurse must also address psychological status and psychiatric issues as one of the key domains to provide a more humane and comprehensive approach for the care of a dying person [5]. This can be achieved by dealing with 4 phases and stages of pathological grief reaction, namely: shock or disbelief, yearning, depression and acceptance. Referral to a patient support group, psychologist, psychiatric services community support while providing psychological support to patient and relatives is necessary.

The need to provide comprehensive assessment and a interdisciplinary plan of care for social needs of the patient and families are a key domain for the provision of more humane and comprehensive approach in the care of a dying person. The sociocultural view of the patient believes that social environment greatly affects life-threatening illness and life-sustaining treatment decisions. Thus, person experiences terminal illness is shaped by broader social and cultural context [5]. Focusing a patient's psychosocial, and sociocultural distress necessity not wait until the very end of life [29]. Palliative care should be placed on sociocultural issue which has revealed a optimistic influence mutually on patient's quality of life and providing peaceful death with dignity [5]. The need to provide culturally-sensitive manner in palliative care is imperative.

Palliative care should be placed on spiritual aspect of care. It must provide comprehensive spiritual support and psychosocial care to enhance the quality of life for people approaching the end of life. Assessing and responding to existential and religious dimensions of the dying patient is important in the provision of spiritual aspect of care and serve as a key domain for the provision of more humane and comprehensive approach in the care of a dying person [5]. In here, the nurse must provide spiritual support by identifying specific religious belief, strengthening one's faith, finding meaning in their lives, attending spiritual needs of patient, providing a sense of caring, providing clergy or spiritual adviser, and allowing prayer and meditation [15]. Thus, restoring spiritual dimension as part of palliative care allows to explore spiritual issues, resolves spiritual distress, provides better spiritual care, promotes spiritual growth [18].

Lastly, family support during end-of-life care in a painful process is important. The need for assessing the patient and family-centered goals of care in caring for terminally ill patients is substantial [30]. Nursing care must focus on provision of family-centered care and family support [15]. Another important nursing role includes establishing a trusting and meaningful relationship with patient and family [30]. 


\section{Primary palliative care skills}

Key palliative care skills are worthwhile in medical and surgical specialties to deliver quality health care for patients with suffering from severe affliction [12]. Palliative care often involves managing symptoms, advance care planning, end-of-life care, supportive care, communication skills $[15,11]$. Such fundamental skills in palliative care are displayed a progressive influence equally on patient's quality of life [23]. The most common core competency for health professionals are interpersonal and communication skills (36\%), shadowed by patient care (24\%) [12].

Interpersonal and communication skills include effective information exchange and association with patients and their families [12]. Palliative care clinicians must provide an open discussion, direct communication, and adequate time so that the dying patient becomes aware and understands about the seriousness of terminal illness, condition and prognosis. Nurse must engage in open discussion about end-of-life issues this can be achieved by giving full disclosure of all treatment possibilities prognosis, and probable outcomes [5]. Nurses are encouraged to talk with family and significant others about their treatment preferences. Beforehand communication about patient worsening and dying requires adequate time for the patient to understand the information and may aid in the consequent choice to initiate end-of-life care [13]. Nurses must be prepared to provide support by using therapeutic communication [15].

Supportive care is for patients with fatal disease aims to optimize patients' health-related quality of life [28]. Supportive management allows enhanced partnership among physicians, nurses, social workers, palliative care experts [25]. The nurse must help the patient and family must have full acceptance of the situation and one's fate. Helps patient understand and balance the benefit versus the burden of treatment principle. The nurse provides ample time for a patient and family to express their fears, ideas, concerns [16]. The nurse must be prepared to encourage family support by showing empathy and genuine concern, offering caring physical presence, allowing grieving process, participating family members in physical care, allowing family says their last goodbyes [15]. The nurse must acknowledge the patient's personal feeling about death, focusing on the patient's needs, teach patient various coping strategies to deal with impending death, be honest and direct in responding to patient's question about death, provide support, active listening.

Nurses should be familiar with patient preference to end-oflife care [19]. Four steps in the end-of-life care: Decision phase (informed consent, patient proxy, family meetings, do-not-resuscitate, aggressive resuscitation, advance care planning, patients' preferences), stabilization phase (symptom management, hydration), near-death (patient care, symptom-relief medications, multidisciplinary, palliative/ hospice care), and bereavement phase (religious activities, bereavement counseling, group session) [28]. The establishment of end-of-life care and bereavement services are integral parts of palliative care delivery [25].

One must meet seriously ill patients' needs for pain management. Pain is commonly referred as the fifth vital sign and must be routinely assessed. Palliative care must provide comprehensive pain control and management to help relieve suffering and to augment the quality of life for people approaching the end of life [5]. Palliative care often involves pain control for patients with chronic, serious, terminal illness and advanced disease. Nurses must be knowledgeable in palliative care to provide effective nursing care in pharmacologic and non-pharmacologic pain management of patients with impending death [16].
One of the fundamental palliative care skills is symptom management, relief and control [11]. Palliative care provides suitable management geared towards symptom relief. Further studies on symptomatology cited that symptom control is an essential and important element in managing patients on supportive care [25]. Symptom relief is an essential goal to attain palliation of difficult symptoms [14]. Symptom management is the cornerstone to ensure the high quality of life and death with dignity. Nurses must perform routine symptom monitoring and assessment of the most commonly reported symptoms during end-of-life [16]. Assessment and management of the clinical symptoms of terminal patients is an issue of increasing concern. Managing symptoms remains the uppermost primacy in hospice palliative care [17]. Appropriate symptom relief must focus the palliative nurse in the treatment of pain, nausea, vomiting, anxiety, depression, restlessness, anorexia, breathlessness, and bowel obstruction [15]. Other distressing symptoms at the endof-life include dyspnea, chronic cough, constipation agitation, delirium [9]. The need for pharmaceutical and drug treatment in the alleviation, management and control of these symptoms is an important principle in patient-centered care. The functional status and patient survival time are two critical concerns in symptom management for terminal patients. Symptom management is an indispensable element of palliative care which incorporates physical, psychological and physiological aspects which represent a holistic method of relief of incurable symptoms at the end stage of life in terminally ill patients [17].

Advance care planning is important because it serves as a foundation for future health care decision making [16]. It must be planned by a healthy competent patient and more exclusively oriented to patients experiencing the predominant decline and extensive disabilities. $[6,25]$. Advance care plan involves determining advance directives, living will, a durable power of attorney, advance medical directive, advance care decisions, use of health proxy, and choosing surrogate [16]. Specifically, it involves considerations of care coordination, a patient centered decision-making, symptom relief and management, and quality of life care. Advance care planning must also include realistic acceptance of death, preference for do-not-resuscitate orders, lifesustaining treatment, structured multidisciplinary therapy, supportive management, comfort measures [25].

The palliative care program commenced as an alternative in the advance care planning (ACP) which includes management benefits, liabilities, an anticipated prognosis and the patient's inclinations were remarkably discussed. Benefit involves hypothetically lifesaving and life-prolonging measures. Prognosis includes condensed life expectancy, impaired QOL, common end-of-life events. Burden from comorbid conditions, symptom distress, treatment and caregiving [14].

\section{The palliative care team}

The elderlies are emerging population shown to demonstrate increasing the complexities of care needs [24]. And it was noted that trainees, caregivers, nurses and physicians feel underprepared to provide end-of-life care skills and lack fundamental training in palliative care [12].

Interdisciplinary, multidisciplinary and inter-professional care team delivered home-based integrated palliative care intervention $[1,29]$. Primary care clinicians provided palliative care to deliver collaborative care management in addressing the unmet palliative needs throughout the illness course of a dying patient [29]. Supportive and collaborative care management of dying patients must involve better collaboration between physicians, nurses, practitioners, social 
workers, pastors/priest/chaplain, and palliative care professionals $[9$, $22,24,25]$. This team also includes a pharmacist, advance practice nurses, gerontologists, a physical therapist, occupational therapists, physiotherapist, psychologist, psychiatrist, dieticians among others $[1,20,22,24,25]$.

Specifically, palliative care nurse roles are patient's advocate, care manager, care coordinator, and case management expert. Physical, occupational, and complementary therapies are useful in assessing and maintaining patient's functional status. Likewise, palliative care is offered along with all other appropriate medical treatments, complimentary therapy, and psychosocial support. Complimentary therapies/medicine are non-pharmacologic interventions for symptom control that include relaxation therapy, guided imagery, massage, acupuncture, aromatherapy, music, and art therapy. Social worker to help patient obtain financial assistance from community programs, medical assistance program, insurance company. On the other hand, pastors, priests and chaplains can provide religious and spiritual counseling services to the dying patient and their family. Overall, social worker referral, support network, community services, and bereavement services are all integral parts of delivery of palliative care [16].

Furthermore, palliative care teams must provide a wide-ranging assessment of the quality-of-life for patients diagnosed with therapeutic difficulties involving advance diseases [21]. All members of the interdisciplinary team must receive proper training to provide the best possible care in the care of older adults [1]. Through this, palliative care specialist can help promote holistic or whole-person centered approach to the discipline [23]. Lastly, all healthcare providers must aim in improving the dying process and improve end-of-life care to allow peaceful death of the patient [5].

\section{Conclusion}

This integrative literature review concluded that there are four general/major themes emerged that is important to palliative care nursing. Such major theme includes basic concepts, palliative care assessment, fundamental palliative care skills, and the palliative care team.

Specialized knowledge, attitude, and skills are all required to provide palliative care to elderly facing life-threatening illness. There is a need for palliative care training in the supportive care of patients with extensive comorbidities [25]. Likewise, the need to develop and explore the training and educational training program for palliative aged care is essentials during the terminal phase of care during the advance process of dying [24]. Palliative care education must also be incorporated in the allied medical science curriculum [25]. There is also limited palliative care certification and formal training programs because generalist practitioners have lack of profound understanding of how biology of aging distresses common illnesses and end-of-life care. Palliative care training can help clinicians recognize the dying patients' preference and goals of care [1]. Palliative care should integrate research and clinical practice in the effective implementation of palliative [29]. Lastly, establishing a specific category for palliative care-related research and publication might be helpful. Supplementary research is indispensable to distinguish and attempt to downgrade the impediments to hospice palliative care research and publication [10].

In general, these are all fundamental mechanisms in bringing highquality patient-centered care predominantly focused to the end-of-life [12].
Overall, this integrative literature review concluded that there are only limited research findings that are currently available about palliative care nursing. Therefore, future research is needed to gather information, further validity and research evidences about this emerging discipline in nursing.

\section{References}

1. Bell SP, Orr NM, Dodson JA, Rich MW, Wenger NK, et al. (2015) What to expect from the evolving field of geriatric cardiology? JACC 66: 1286-1299.

2. Berman A, Snyder S, Kozier B, Erb G (2008) Kozier\& Erb's fundamentals of nursing: concepts, process and practice.8th edtn. Singapore: Pearson Education South Asia Pte. Ltd.

3. Bloomer MJ, Mari Botti M, Runacrese F, Poone P, Barnfield J, et al. (2018) End-of-life care for older people in subacute care: A retrospective clinical audit. Australia.

4. Brannstrom M, Boman K (2014) Effects of personcentred and integrated chronic hear failure and palliative home care: PREFER: a randomized controlled study. Eur J Heart Fail 16.

5. Bridge DT (2008) Curing diseases and healing suffering: Inspiration from developments in palliative medicine. Int J Gerontol 2: 29-32.

6. Carmody S, Forster S (2003) Aged care nursing: a guide to practice. Australia: Ausmed Publications Pty. Ltd 176-180.

7. Carrasco JM, Lynch TJ, Garralda E, Woitha K, Elsner F, et al. (2015) palliative care medical education in european universities: a descriptive study and numerical scoring system proposal for assessing educational development. J Pain Symptom Manag 50: 516-526.

8. Chao CT, Tsai HB, Shih CY, Hsu SH, Hung YC, et al. (2016) Establishment of a renal supportive care program: Experience from a rural community hospital in Taiwan. $J$ Formos Med Assoc 115: 490-500.

9. Chan CK, Wong SH, Ho EL, Cheng YK, Lam WO, et al. (2010) Supportive managemen in patients with end-stage renal disease: Local experience in Hong Kong. Hong Kong J Nephrol 12: 31-36.

10. Chang HT, Lin MH, Chen CK, Hwang SJ, Hwang IH, et al. (2016) Hospice palliative care article publications: An analysis of the web of science database from 1993 to 2013. $J$ Chin Med Assoc 79: 29-33.

11. Chen CY (2012) Principles of patient-centered care in control of terminal symptoms. $J C G G$ 3: $87-88$.

12. Gale M, Kawai F, Pan C, Morris J, Gasperino J (2014) Withdrawal of mechanical ventilation: A systematic review and approach following two unusual cases. New York: American College of Chest Physicians, Elsevier Inc.

13. Garcia JB, Rodrigues RF, LimaSF (2014) Structuring a palliative care services in Brazil: experience report. Brazilian Society of Anesthesiology 64: 286-291.

14. Harris JA, Herrel LA, Healy MA, Wancata LM, Perumalswami CR (2016) Milestone for the final mile: Interdisciplinary distinctions in primary palliative care skills training. Journal of Pain and Symptom Management 52: 345-352.

15. Hegarty M, Currow DC (2007) Palliative aged care: Collaborative partnerships between gerontology, geriatrics and palliative care. Int J Gerontol 1: 112-117.

16. Hopp FP, Zalenski RJ, Waselewsky D, Burn J, Camp J, et al. (2016) Results of a hospital based palliative care intervention for patients with an acute exacerbation of chronic heart failure. J Card Fail 22: 1033-1036.

17. Kavalieratos D, Gelfman LP, Tycon LE (2017) palliative care in heart failure rationale evidence, and future priorities. $\mathrm{J} \mathrm{Am} \mathrm{Coll} \mathrm{J} \mathrm{Am} \mathrm{Coll} \mathrm{Cardiol} \mathrm{65:} \mathrm{750-753.}$

18. Monahan S, Neighbors M, and Green. Phipps' medical-surgical nursing: Health and illness perspective.

19. Mendoza JM (2015) competencies in palliative care for cardiology fellows. $J$ Am Coll $J$ Am Coll Cardiol 65: 750-753.

20. Morton PG, Fontaine DK (2009) Critical care nursing: A holistic approach. 9th edition Philadelphia: Lippincott William and Wilkins. A Wolter Kluwer Co.

21. Nicholl C, Wilson KJ, Webster S (2008) Elderly care medicine. 7th edition. Massachusetts: Blackwell Publishing.

22. Rich MW, Chyun DA (2016) Knowledge gaps in cardiovascular care of the older adult population. J Am Coll J Am Coll Cardiol 67: 2419-2440. 
23. Rogers JG, Patel CB, Mentz RJ, Granger BB, Steinhauser KE et al. (2017) Palliative care in heart failure: the PAL-HF randomized, controlled clinical trial. $J$ Am Coll Cardiol 70: 331-341.

24. Saunders MJ, Yeh CK, Hou LT, Katz MS (2005) Geriatric medical education and training in the United States. J Chin Med Assoc 68: 547-556. [Crossref]

25. Sidebottom AC, Jorgenson A, Richards H, Kirven J, Sillah A (2015) Inpatient palliative care for patients with acute heart failure: outcomes from a randomized trial. $J$ Palliat Med 18: 134-142.

26. Smeltzer SC, Bare BG, Hinkle JL, Cheever KH (2009) Brunner \& Suddarth's textbook of medical-surgical nursing. 12th edition. Philadelphia: Lippincott William and Wilkins.
27. Struckmann V, Leijten FR, Ginneken EV, Kraus M, Reiss M, et al. (2018) Relevant models and elements of integrated care for multi-morbidity: Results of a scoping review. Health Policy 122: 23-35.

28. Taylor C, Lillis C, LeMone P (2005) Fundamentals of nursing: The art and science of nursing care. Philadelphia: Lippincott William and Wilkins.

29. Tse DW (2009) experience of a renal palliative care program in a Hong Kong Center: characteristics of patients who prefer palliative care to dialysis. Hong Kong J Nephrol 2: $50-58$.

30. Weiss BD, Mohler MJ, Fain MJ (2011) Geriatrics in Taiwan: What is the solution? JCGG 2: 93-95.

31. Wu CH, Chang CI, Chen CY (2012) Overview of studies related to geriatric syndrome in Taiwan. JCGG 3: 14-20.

Copyright: ( 92019 Guerrero JG. This is an open-access article distributed under the terms of the Creative Commons Attribution License, which permits unrestricted use, distribution, and reproduction in any medium, provided the original author and source are credited. 